\title{
Aircraft 4D Trajectory Prediction in Civil Aviation: A Review
}

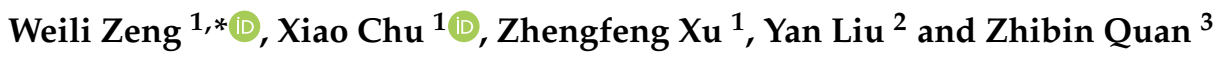 \\ 1 College of Civil Aviation, Nanjing University of Aeronautics and Astronautics, No. 29 General Avenue, \\ Nanjing 211106, China; chuxiao0401@nuaa.edu.cn (X.C.); xuzhengfeng@nuaa.edu.cn (Z.X.) \\ 2 State Key Laboratory of Air Traffic Management System and Technology, Nanjing 211106, China; \\ liuyan@163.com \\ 3 School of Automation, Southeast University, Nanjing 210096, China; sigma_quan@seu.edu.cn \\ * Correspondence: zwlnuaa@nuaa.edu.cn
}

check for

updates

Citation: Zeng, W.; Chu, X.; Xu, Z.;

Liu, Y.; Quan, Z. Aircraft 4D

Trajectory Prediction in Civil

Aviation: A Review. Aerospace 2022, 9,

91. https://doi.org/10.3390/

aerospace 9020091

Academic Editor: Manuel Soler

Received: 30 November 2021

Accepted: 7 February 2022

Published: 10 February 2022

Publisher's Note: MDPI stays neutral with regard to jurisdictional claims in published maps and institutional affiliations.

Copyright: () 2022 by the authors Licensee MDPI, Basel, Switzerland. This article is an open access article distributed under the terms and conditions of the Creative Commons Attribution (CC BY) license (https:// creativecommons.org/licenses/by/ $4.0 /)$.

\begin{abstract}
Aircraft four dimensional (4D, including longitude, latitude, altitude and time) trajectory prediction is a key technology for existing automation systems and the basis for future trajectorybased operations. This paper firstly summarizes the background and significance of the trajectory prediction problems and then introduces the definition and basic process of trajectory prediction, including four modules: preparation, prediction, update, and output. In addition, the trajectory prediction methods are summarized into three types: the state estimation model, the Kinetic model, and the machine learning model, and in-depth analysis of various models is carried out. Further, the relevant databases required for the study are introduced, including the aircraft performance database, aircraft monitoring database, and meteorological database. Finally, challenges and future development directions of the current trajectory prediction problem are summarized.
\end{abstract}

Keywords: aircraft trajectory prediction; 4D trajectory; civil aviation; review; machine learning

\section{Introduction}

With the rapid economic development, the demand for air transport continues to grow rapidly, and the contradiction between aviation demand and airspace capacity has become increasingly prominent. It is predicted that in the next 20 years, the annual growth rate of global air transportation will be about $4.4 \%$, and the volume of air traffic in China will increase by 3.5 times [1], which brings major challenges to the development of the civil aviation industry. At present, the operation mode of the fixed airspace sector and the route has problems such as structural solidification, cascading failures, and limited capacity, which not only restrict the space for air communication optimization but also cannot support future trajectory-based and performance-based airspace operation modes.

In order to continue to meet the different expectations of various stakeholders in terms of the comprehensive performance of operational efficiency, flight safety, cost-effectiveness, and environmental impact, many countries and organizations have initiated various types of air traffic system upgrade projects to cope with the ever-growing demand for air traffic, complex air traffic systems, and diverse operating environments [2]. Whether it is the current sector-based operation mode or the future trajectory-based operation mode, the prediction of the future trajectory of the aircraft is the key to the efficient operation of these two modes. At the same time, accurate trajectory prediction is the basis for decision-making systems such as arrival and departure sequencing, conflict detection, airspace situational awareness, and flight flow management, which can greatly reduce the uncertainty of an aircraft's future flight and improve the predictability of air traffic [3,4].

In view of the important status of trajectory prediction, accurate trajectory prediction is a relatively systematic and relatively difficult task, and the influence of various deterministic and uncertain factors needs to be considered at the same time. With the rapid development of machine learning and data mining technology in recent years, many new data-driven 
trajectory prediction methods have emerged, updating traditional dynamics and other methods. Therefore, it is necessary to comprehensively sort out and summarize the current research status of trajectory prediction to lay the foundation for relevant researchers to carry out the following trajectory prediction research. The purpose of this article is to review the classic method of trajectory prediction and sort out its latest developments and applications in the field of air traffic, allowing relevant researchers to have an overall grasp of the development of trajectory prediction and providing a new direction for future research on trajectory prediction.

The organization of the paper is as follows. Section 2 introduces the trajectory prediction problem and related definitions. Section 3 elaborates on the entire process of trajectory prediction in detail. Section 4 begins to comprehensively sort out and summarize the methods of trajectory prediction. Section 5 introduces several evaluation indexes of the track prediction effect. Section 6 introduces the available databases for trajectory prediction, providing data sources for relevant researchers to carry out research. Finally, Section 7 looks forward to the challenges faced by trajectory prediction and new directions that need further research in the future.

\section{Problem Description and Definition}

The Federal Aviation Administration (FAA)/Eurocontrol Action Plan 16 (AP16) defines trajectory as a four-dimensional (latitude, longitude, altitude, and time) description of an aircraft flight path [5,6]. The International Civil Aviation Organization (ICAO) extended the definition to the ground operation and expressed the trajectory as the description of aircraft motion in the air and on the ground, including position, time, speed, acceleration, and other attributes [7].

Due to airspace congestion, weather, temporary military activities, airspace restrictions, etc., the actual flight process of aircraft sometimes does not follow the route planned in advance, as shown in Figure 1. This reduces the predictability of air traffic, increases the difficulty of air traffic management, and seriously hinders the efficient operation of the air traffic management system. Therefore, it is necessary to predict the four-dimensional trajectory of the aircraft.

The trajectory prediction is the process of estimating the future states of the aircraft based on the current aircraft state, estimation of the pilot and controller intent, expected environmental conditions, and computer models of aircraft performance and procedures [6]. According to whether the aircraft takes off, it can be divided into strategic prediction and tactical prediction. Strategic forecasting mainly predicts the possible future flight trajectories of the aircraft based on the aircraft's flight plan, weather forecast information, aircraft performance, historical flight patterns, and other information. The tactical prediction adds more dynamic information, such as the aircraft's own flight status information, airspace congestion, and so on.

In terms of the time scale, the trajectory prediction can be divided into two categories [8,9]: (1) Short-term prediction: short-term prediction in a few minutes or less. Since the forecast interval is small, there is no need to know long-term intentions and weather, but during the duration of the forecast interval, additional assumptions (such as fixed aircraft control and constant turn rate) are required. Since these assumptions are indeed valid in a smaller propagation interval, the prediction accuracy increases as the size of the prediction interval decreases. Short-term prediction allows the immediate risk of conflict to be detected, thus providing the possibility to resolve the real conflict. At the same time, a good TP can promote the generation of good alternative trajectories, compatible with existing constraints. (2) Medium- and long-term forecasts: long-term forecasts of ten minutes or more. Due to the large prediction interval, it is necessary to use information such as long-term intentions, environmental data, aircraft performance data, and navigation data. However, the uncertainty of this information will cause the prediction accuracy to decrease as the prediction time interval increases. Mid-to-long-term forecasting is helpful for effective planning and management and the regular assessment of airspace operation 
status and is mainly used for airspace flow assessment and the formulation of the flight plan of the aircraft operation center (or flight operation center).

According to the form of the prediction result, the trajectory prediction can be divided into two other categories [10]: (1) Deterministic prediction: This is composed of the nominal method and the worst-case method and generally directly outputs the predicted fourdimensional trajectory information [11]. The nominal method cannot perfectly describe the uncertainty of the aircraft's future behavior. Therefore, as the forecasting time increases, its accuracy may decrease. The worst-case method usually assumes that an airplane will perform any one of a set of maneuvers and considers the worst-case aircraft trajectory prediction. This method is conservative. (2) Probabilistic prediction: This describes the potential changes of the aircraft's future trajectory through modeling uncertainty, using the probability density function to describe the aircraft trajectory, which can provide more accurate long-term predictions than deterministic methods.

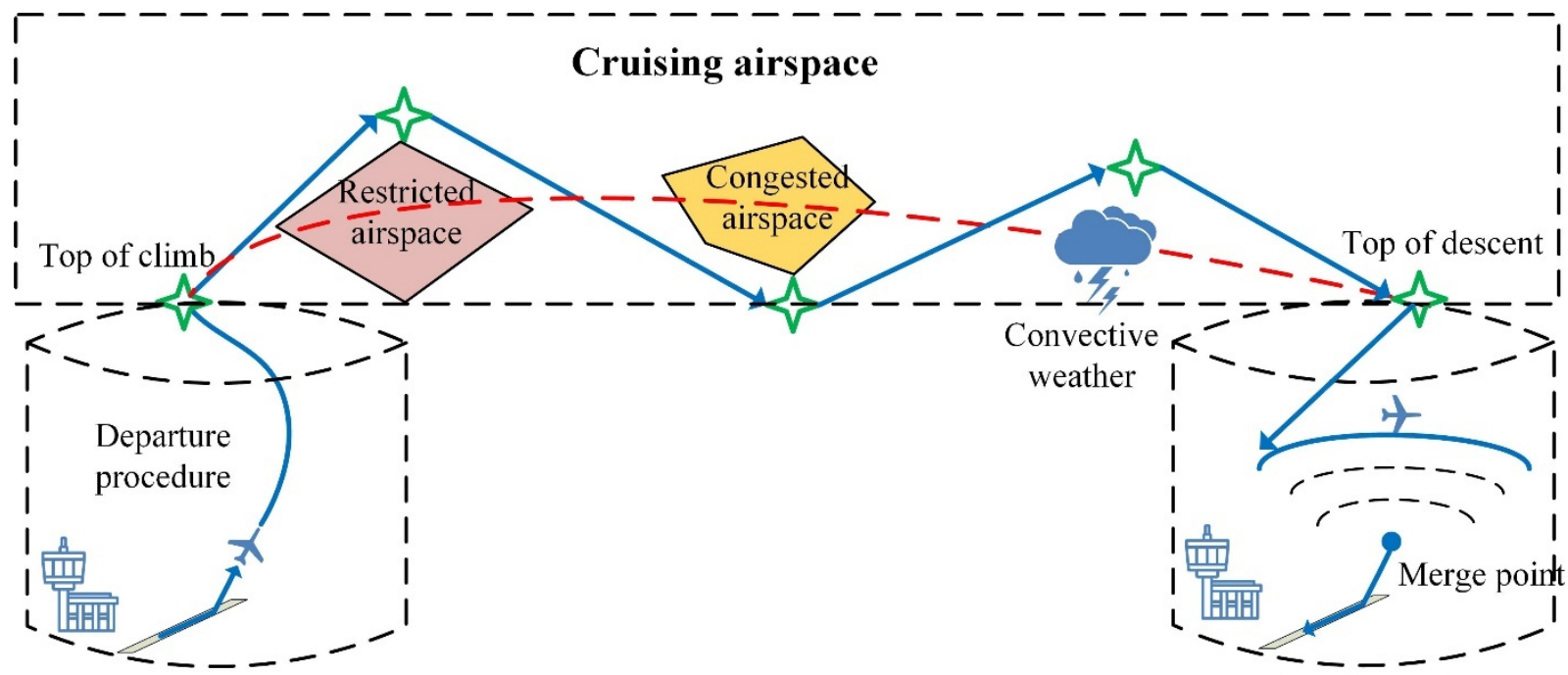

Departure Airport

---- Planned route

Actual flight route

Destination airport

Figure 1. Schematic diagram of an aircraft in different stages of flight.

\section{Trajectory Prediction Process}

Trajectory prediction is usually performed by trajectory predictors (TPs). Because different DST and automation systems have very different requirements for TPs in terms of accuracy, uncertainty, response time, and input data, the structure, process, function, and performance of TPs depend entirely on the application of trajectory prediction [12]. This will lead to the coexistence of multiple completely different TPs in the ATM system, which brings potential problems to the interoperability of different automation systems in the air and on the ground. For the purpose of ATM interoperability, AP16 proposed a general TP model [5].

Figure 2 shows the general trajectory prediction process introduced in AP16, which includes four modules: preparation, prediction, update, and output. Here it is further broken down into the specific steps described in the FAA's previous research management plan on general trajectory modeling [5]. The input data of the preparation process include flight plans, airline operating procedures, air traffic control (ATC) restrictions on weather, and aircraft performance, etc. The process will integrate these data to establish a flight script (Flight Script, FS) to describe the predicted flight segment and construct a behavioral model. A behavior model is an ordered list of maneuvers that the aircraft plans to execute (also known as flight intent), describing how to operate the aircraft to meet trajectory constraints and user preferences in a clear way [13]. The prediction process is the core process of TP. It uses a set of methods and algorithms implemented by the trajectory engine 
(TE) to obtain a calculated trajectory (CT) by combining behavior models, meteorological data, and aircraft performance data. The update process updates the flight script according to the constantly changing information, which can be performed through a regular update or monitoring of the consistency between the prediction and the true value, which may lead to the generation of a new flight script or the modification of information and trigger a new preparation process. The output process exports the output data of the TP to the client application, including the predicted trajectory and error and warning messages that notify the client of the availability and/or quality of the output data.

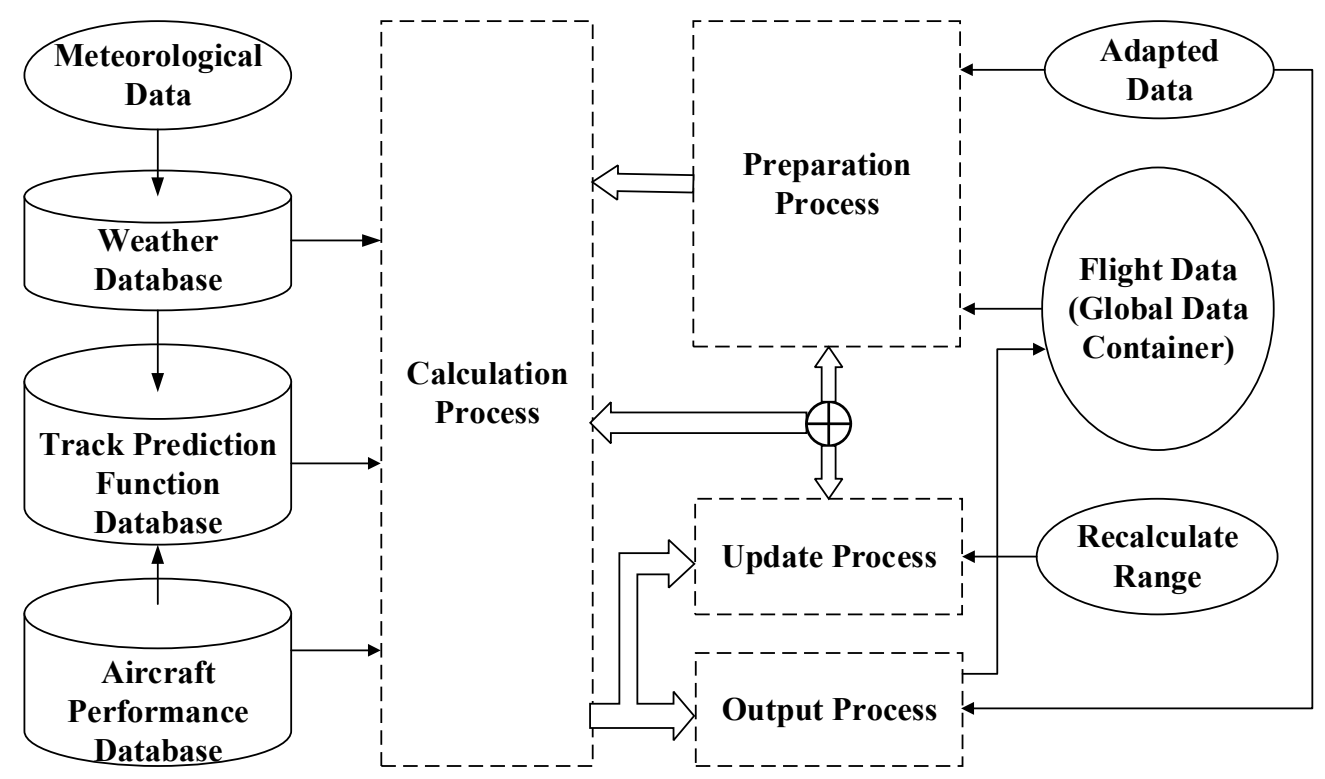

Figure 2. General TP process.

\section{Prediction Methods}

This paper divides the trajectory prediction methods into state estimation methods, kinetic methods, and machine learning methods.

The state estimation model only establishes the motion equation based on the aircraft's position, speed, acceleration, and other attributes, so as to realize the propagation of the estimate. The model is relatively simple, but because it cannot accurately capture the aircraft's maneuvering uncertainty for a long time, it will cause large errors. Therefore, it can only work in a short time. Although flight intention information has been incorporated into the prediction model in order to improve the prediction accuracy, the inference of the intention is only relatively accurate in the short term.

Although the kinetic model is analyzed from the perspective of aircraft forces, in order to simplify the model, most of it is realized under some ideal assumptions, with little consideration of actual constraints and human behavior [14-19]; in addition, due to the consideration of aircraft performance, aircraft status, environmental conditions, and aircraft intentions, the model requires a large number of parameters, some of which are commercially sensitive and not easy to obtain, and the other part uses predefined settings or estimates in the existing database. Information is often not accurate enough. Thereby, the research that is being conducted by the projects PJ31 DIGITS and PJ38 ADSCENSIO in SESAR is committed to improving this situation. Meanwhile, the PCP Project on Initial Trajectory Sharing is also ongoing, and there is still a long way to go if they are promoted globally, so once the data resources are limited or not fully supported, the prediction accuracy of the model will be greatly reduced or even not applicable. The uncertainty of these input data sources will obviously bring greater uncertainty to the trajectory prediction. These errors include modeling errors, initial conditions errors, aircraft-specific errors, environmental information errors, and intention errors. 
Machine learning models use machine learning and data mining algorithms to learn historical flight trajectories and meteorological data to predict flight trajectories. They are constructed under weak or even no assumptions. The machine learning model does not require explicit modeling of aircraft performance, procedures, and airspace, that is, it does not need to have a deep understanding of the relationship between the force and motion of the aircraft and only needs to learn the laws from massive data. Therefore, the machine learning model is actually a kind of data engineering in nature, and the larger the amount of data, the better the effect. Due to a large amount of flight trajectory data available, it makes it possible to mine complex trajectory patterns and extract important features, which provides data and a preliminary basis for trajectory prediction.

\subsection{State Estimation Model}

In practical applications, we can regard the operating process of a physical system as a state transition process. The trajectory prediction estimates the position, speed, and other states generated during the flight of the aircraft, and the state estimation model uses the state space theory to mathematically model the physical system. Any nonlinear system that can be described by a state-space model can use it to model the physical system. It is widely used in the field of target tracking and has become a class of methods for studying track prediction problems. The state estimation model needs to construct the state transition matrix in the state equation through the equation of motion and study the relationship between position, historical position, speed, acceleration, angle, and other states at each time point in the future. According to different assumptions about whether the aircraft has a single flight mode or multiple modes in the prediction process, this type of method is divided into single model estimation and multi-model estimation. Table 1 summarizes the various methods of the state estimation model introduced in this section.

\subsubsection{Single Model Estimation}

In order to simplify the model, the multi-mode nature of aircraft motion is ignored, and the state estimation is performed under the assumption of a single mode. The most classic method in single model estimation is the Kalman Filter (KF) algorithm [20,21]. KF estimates the state of the system at the next moment according to the state equation and observation equation of the system [9].

$$
\left\{\begin{array}{c}
X(t+1)=\Phi(t) X(t)+\zeta_{X}(t) \\
Z(t)=H(t) X(t)+\zeta_{Z}(t)
\end{array}\right.
$$

where $X(t)$ is the state of the aircraft at time $t$ (including position, velocity, acceleration, angular velocity, etc.), and it is also the input of the model, that is, the state at time $t$ is known, and the state $X(t+1)$ at time $t+1$ is predicted, which is the output of the model; $\Phi(t)$ represents the state transition matrix; $\zeta_{X}(t)$ is the zero-mean Gaussian white noise sequence representing process noise, and its covariance matrix is $Q(t) ; Z(t)$ is the observation vector, representing the observation value of the aircraft at moment $t$ (the information to be predicted, such as position and time, etc.); $H(t)$ is the observation matrix that links the state with the observation value; $\zeta_{Z}(t)$ represents the observation noise vector generated during the movement, and is also assumed to be zero-mean Gaussian white noise, and its covariance matrix is $R(t)$. When the kinematic equations are used to construct $\Phi(t-1)$ and $H(t)$, the state equations and observation equations are obtained accordingly.

Based on the state equation and observation equation discussed above, the KF algorithm is used to calculate the unbiased minimum variance estimate of the state. In addition to state estimation, the filter also generates a covariance matrix of state estimation errors. KF is divided into two steps: observation update and process update. Observation update improves state estimation based on new observations, as shown in Formulas (2)-(3).

$$
\widetilde{X}(t)=\hat{X}(t)+K(t)[Z(t)-H(t) \hat{X}(t)]
$$




$$
\widetilde{P}(t)=[I-K(t) H(t)] P(t)
$$

Among them, $I$ represents the identity matrix, $\widetilde{X}(t)$ represents the improvement of state estimation, $\widetilde{P}(t)$ represents the improvement of error covariance, and $K(t)$ represents the Kalman gain matrix. The calculation method is as follows:

$$
K(t)=P(t) H^{T}(t)\left[H(t) P(t) H^{T}(t)+R(t)\right]^{-1}
$$

The process update propagates the state estimation and error covariance according to the state equation until the observation value at the next moment is obtained.

Before using KF for state estimation, the state vector $\hat{X}(t)$ and its error covariance matrix $P(t)$ must be initialized. In addition, the process noise covariance matrix $Q(t)$ and the observation noise covariance matrix $R(t)$ must also be selected. The choice of $Q(t)$ and $R(t)$ determines the required balance between smoothness and fast response.

At present, the KF algorithm [9,22], particle filter algorithm [23], hidden Markov model (HMM) [24-27], and their various improved algorithms [28] are all applied in the trajectory prediction problem. Chatterji [9] used KF for short-term trajectory prediction, using the current ground speed and trajectory angle estimation and kinematic equations to propagate the current position estimate forward to obtain the position estimate at the future time. As the intention of the aircraft is assumed to be fixed in the short term, when the aircraft deviates significantly from the assumed intention, the prediction accuracy will be affected. In addition to using the KF algorithm, Lymperopoulos et al. [23] proved the inefficiency of several sequential Monte Carlo algorithms in the high-dimensional state estimation problem involving multiple aircraft and thus proposed a new particle filter algorithm. Another state estimation method commonly used is HMM. Ayhan et al. [26] applied HMM to predict trajectories considering environmental uncertainty and learned the correlation between historical trajectories and relevant weather parameters.

\subsubsection{Multi-Model Estimation}

Although a single model has been widely used and has achieved certain effects, a single model cannot well estimate the hybrid system with different modes, and the aircraft trajectory prediction problem can be regarded as a stochastic linear hybrid system (stochastic linear hybrid system, SLHS) estimation problem, which needs to be solved by a multi-model method. When using SLHS to simulate aircraft motion, it is divided into many flight modes, such as constant speed, coordinated turning, constant descent, etc. In each flight mode, simpler dynamic equations can be used to describe the aircraft motion model. For different flight modes, a multi-model estimation can achieve superior performance by using different state estimators that match the aircraft motion model. However, the multi-model algorithm is an important method to solve the SLHS estimation problem, and its computational cost increases exponentially with time. Therefore, sub-optimal algorithms such as the generalized pseudo-Bayes algorithm and interacting multiple model (IMM) are proposed. The IMM algorithm has excellent performance and low computational cost and has been successfully applied to track prediction. Song et al. [3] used IMM for state and model estimation and updated the flight mode transition probability matrix by using a data mining algorithm to extract a typical trajectory library as intent information to achieve state update.

The multi-model algorithm models the flight mode transition as a Markov process with a constant mode transition probability matrix, independent of continuous state variables [29]. However, in order to ensure flight safety and facilitate air traffic management, aircraft generally follow a flight plan composed of a fixed route structure, so the behavior of the aircraft is composed of discrete transitions between many flight modes (discrete states) and continuous motion corresponding to specific flight modes (continuous states). Therefore, based on the information provided by the archived flight plan, many documents model the transition probability of flight mode as dependent on the continuous state of the aircraft (such as position, speed, etc.) [30-33]. Seah et al. [31] considered that the flight 
mode transition probability depends on the continuous state of the aircraft and no longer modeled the mode transition as a Markov process and proposed a multi-model Kalman filter algorithm based on the continuous state correlation mode transition matrix to solve the aircraft tracking problem.

In addition, the standard IMM algorithm assumes that the residual error is zero mean and calculates the probability of mode transition through the likelihood function. Due to the incompleteness of the pattern set in the IMM algorithm, this assumption is usually invalid [34]. Therefore, many researchers have proposed improved multi-model estimation methods for this [35-38], among which Yepes et al. [36] proposed an intention-based trajectory prediction algorithm, inferring the intention of the aircraft that is most likely to represent the actual situation, and calculating the nominal trajectory prediction based on the intention information based on the state estimation and flight mode estimation provided by the residual mean IMM algorithm.

Table 1. Overview of state estimation model methods.

\begin{tabular}{cc}
\hline Single model estimation & KF: [9,20,22] \\
& Particle filter algorithm: [23] \\
HMM: [24-27] \\
\hline Multi-model estimation & Multi-model KF: [31-33] \\
& IMM: [3,29,39] \\
& Improved IMM: [35-38] \\
\hline
\end{tabular}

\subsection{Kinetic Model}

The kinetic-based trajectory prediction model mainly studies the relationship between the force acting on the aircraft and the aircraft movement and also involves the force and movement of the aircraft. The dynamic model is expressed as a set of differential equations, given the current state of the aircraft (such as mass, thrust, drag, position, speed, angle of inclination), meteorological conditions (such as wind speed and direction), and aircraft intentions (such as target speed or climb rate), through the integral-differential equation in a time interval to predict the continuous points of the future aircraft trajectory [40]. Therefore, this method integrates aircraft intent, performance parameters, and meteorological environment data for calculation.

Here, we first introduce the point mass model (PMM) commonly used in kinetic models [12,41], as shown in Figure 3. The model describes the relationships between the force acting on the aircraft (including the gravity $m g$, engine thrust $T$, aerodynamic lift $L$, and drag $D$ ) and the state of the aircraft (including horizontal position $(x, y)$, altitude $h$, true airspeed $V_{\text {TAS }}$, path angle $\gamma$, heading angle $\psi$ and bank angle $\varphi$ ). Some simplified assumptions are made from the perspective of ATM, assuming that the thrust and drag vectors are collinear with the airspeed vectors, and the lift is perpendicular to these vectors (that is $\alpha=\beta=0$ ), ignoring fast dynamics, and treating $\gamma, T$ and $\varphi$ as inputs. Therefore, the PMM of aircraft movement is shown in Formulas (5)-(10); $\eta$ is the thrust ratio fuel consumption parameter, and $w_{1}$ and $w_{2}$ are the easterly and northerly wind velocity components.

$$
\begin{gathered}
\dot{x}=V_{\text {TAS }} \sin (\psi) \cos (\gamma)+w_{1} \\
\dot{y}=V_{\text {TAS }} \cos (\psi) \cos (\gamma)+w_{2} \\
\dot{h}=V_{\text {TAS }} \sin (\gamma) \\
\dot{V}_{\mathrm{TAS}}=(T-D) / m-g \sin (\gamma) \\
\dot{\psi}=g \tan \varphi / V_{\mathrm{TAS}} \\
\dot{m}=-\eta T
\end{gathered}
$$

Predicting the trajectory requires a model of the aerodynamic drag of any airframe flying in the air at a given speed. In addition, the maximum climb thrust is also required, 
which depends on the engine that the aircraft is equipped with. If the initial state of the aircraft (mass, position, speed, etc.) is not known in advance and the pilot's intentions of how to operate the aircraft in the future (the law of thrust, the law of speed, or the rate of climb) are not known in advance, predictions cannot be made. When the aircraft is operating at a given calibrated airspeed or Mach number, the calculation $V_{T A S}$ requires knowledge of the atmospheric conditions (air temperature and pressure). Finally, there is a need to predict the trajectory on the ground, not only through the air, but also the magnitude and direction of the wind.

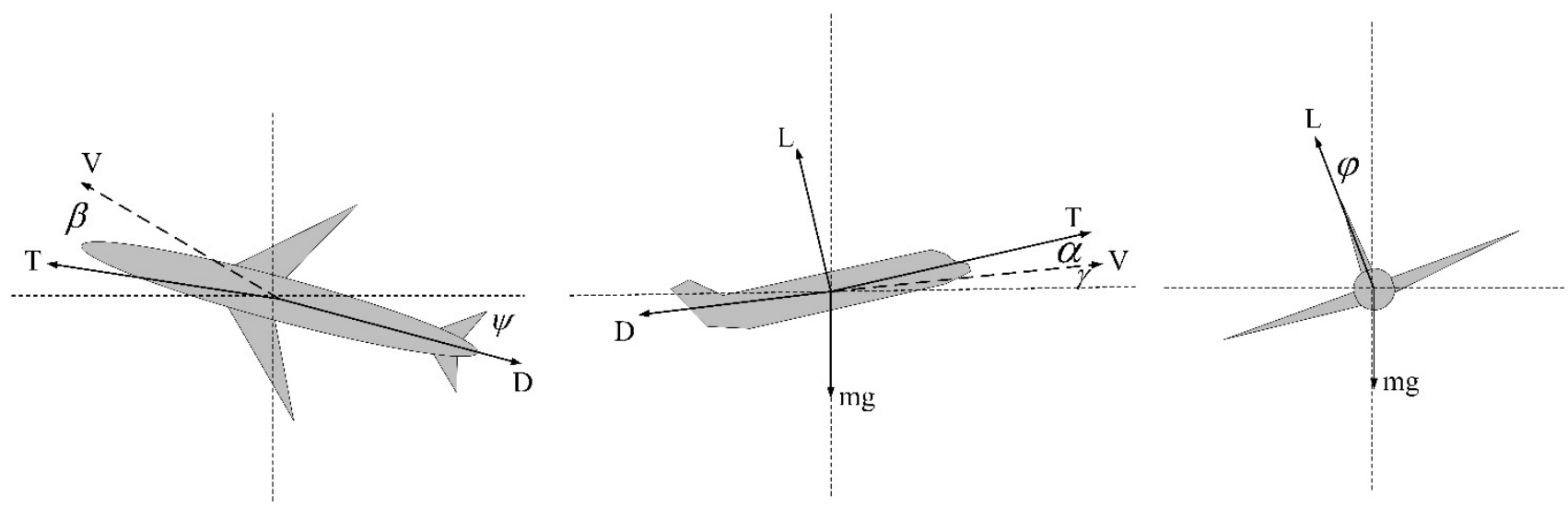

Figure 3. The free-body diagram of forces acting on the point mass.

PMM (sometimes called the total energy model) is currently the most widely used. PMM first defines a non-inertial reference coordinate system that is easy to coordinate with the aircraft force and determines the aircraft acceleration in the coordinate system and then uses Newton's second law (the power of the force acting on the aircraft is equal to the rate of increase of potential energy and kinetic energy) to derive the dynamic equations, finally combining the kinematic and dynamic equations to derive the EOM composed of a set of differential equations, such as the full six-degrees-of-freedom EOM. Through some simplified assumptions, the motion of the aircraft can be reduced to fewer degrees of freedom [42]. PMM can be used for aircraft motion modeling in a fast simulation environment $[12,43,44]$. Fukuda et al. [44] used the total energy model to model the motion of the aircraft and equated the work rate of the force acting on the aircraft with the rate of increase in potential energy and kinetic energy.

Since the motion of the aircraft is an SLHS with different flight modes, it is more reasonable to establish corresponding motion equations based on different flight modes. Therefore, many scholars use PMM to make predictions under SLHS [14,45,46]. Lymperopoulos et al. [14] used the point mass model to model the aircraft under the stochastic hybrid system, combining the continuous state from the physical movement of the aircraft and the discrete state from the flight plan and the flight management system (FMS). Lee et al. [46] proposed an aircraft tracking and estimated time of arrival prediction algorithm based on a stochastic hybrid system model, deduced a nonlinear dynamics model of the continuous motion of the aircraft in each flight mode, and used the continuous state transition probability to model the discrete transition between flight modes.

Unified and comprehensive intention information is necessary for trajectory prediction. At present, related researchers have proposed the expansion and improvement of flight scripts and are committed to providing a formal aircraft intention description language [15,16,18,47-49]. Aircraft intentions consist of a set of structured instructions that are used by the trajectory calculation infrastructure to provide a clear trajectory, which can be considered an abstraction of the way the pilot behaves and/or FMS command aircraft behavior. The determination of the intent needs to be combined with the flight intent (such as following the instructions of standard terminal arrival procedures or standard departure procedures), the operating preferences of the airline, and the actual pilot's decision-making 
process [47]. Therefore, the parameters required by aircraft intentions mainly come from the airline operating data and navigation data [44]. Airline operating data include the preferred altitude, speed, and mass during the climb, cruise, and descent phases. The navigation database provides relevant information such as airports, runways, and waypoint locations. Félix et al. [47] provided a computerized method for expressing aircraft intentions using formal language, which combined the intention information with standard operating procedures, airline's operating preferences, and actual pilot's decision-making process and integrated the initial state, aircraft intention description, aircraft performance model, and environment model to design a trajectory prediction engine.

Aircraft performance parameters provide the values of aircraft performance required by the dynamics model. These values depend on the type of aircraft whose trajectory is being calculated, the current state of motion of the aircraft (position, speed, mass, etc.), and current atmospheric conditions. In addition, it may also depend on the aircraft's intent. Therefore, performance parameters mainly come from flight performance data, monitoring data, aircraft intent, and environmental status information. In view of the difficulty of obtaining performance parameters, related research has appeared [50-55]. The base of aircraft data (BADA) is an aircraft performance database based on the kinetic approach to aircraft performance modeling that has been developed and maintained by the Eurocontrol Experimental Centre (EEC). The information provided in BADA is designed for use in trajectory simulation and prediction in ATM research as well as for modeling and strategic planning in-ground ATM operations. Alligier [53] improved the trajectory prediction accuracy by learning some of the unknown point-mass model parameters from past observations; the performances of the proposed method are compared with the results of standard model-based methods relying on the Eurocontrol Base of Aircraft Data (BADA). Thipphavong et al. [50] proposed a universal real-time adaptive weighting algorithm to improve the accuracy of climb trajectory prediction. The weight of the aircraft in the model is dynamically adjusted through the available radar trajectory and weather data, without any additional data from the aviation operation center or the aircraft data. Baklacioglu et al. [51] used genetic algorithms (GAs) to derive a new aviation propulsion model (APM) from the flight manual data of transport aircraft for accurate trajectory prediction. This new genetic algorithm-based APM has made some improvements to existing models. The use of gas improved the accuracy of propulsion and aerodynamic modeling. Sun [54] used various data mining methods, as well as a maximum likelihood estimation approach, to generate parametric models for these performance parameters. All parametric models combined can be used to describe a complete flight. Sun [55] combined the fuel flow model, the total energy model, and the reference model to independently calculate different initial aircraft masses and then used a Bayesian approach to generate a maximum a posteriori estimate of the initial aircraft mass. Alligier [56] improved the trajectory prediction accuracy by predicting some of the unknown point-mass model parameters using a stochastic gradient-boosting tree algorithm.

\subsection{Machine Learning Model}

Except for some irregularities, each execution of the flight in the historical data usually follows the same planned route and flies over the same waypoint sequence, which proves that the historical trajectories have certain regularity, providing the feasibility for using machine learning to solve the problem of track prediction [27]. This type of method mines the law of aircraft trajectory changes over time from a large amount of data and uses the law to predict the position trajectory. On the one hand, it mainly relies on the underlying laws of aircraft operation and excavates representative trajectory patterns. On the other hand, it is based on the reconstruction of input and output space [12]. Here, it is divided into regression models, neural networks, and other methods. Table 2 summarizes the methods commonly used by scholars in the past when using machine learning models. 


\subsubsection{Regression Model}

Here, we first introduce the basic model in the regression model-multiple linear regression, which is generally defined as:

$$
y=\beta_{0}+\beta_{1} x_{1}+\cdots+\beta_{m} x_{m}+\varepsilon
$$

$y$ is the dependent variable; it refers to the predicted value of the track, such as the three-dimensional position and time information of the track; $x_{i}$ is the independent variable whose optional features include the historical position, time, speed, heading, the rate of decline, and other information of the aircraft, $i=1, \cdots, m, \beta_{j}$ is the parameter, $j=0, \cdots, m, \varepsilon$ is the error assuming that the mean is zero.

The linear least squares estimator is defined as:

$$
\hat{y}=\hat{\beta}_{0}+\hat{\beta}_{1} x_{1}+\cdots+\hat{\beta}_{m} x_{m}
$$

$\hat{y}$ is the estimated value of $y, \hat{\beta}_{j}$ is the estimated value of $\beta_{j}, j=0, \cdots, m$; in the linear least-squares method, the parameter $\hat{\beta}_{j}$ is obtained by minimizing the residual sum of squares, that is, when $n$ sets of observations $\left\{y_{i}, x_{i 1}, \cdots, x_{i m}\right\}$ are given, $i=1, \cdots, n$, The function that needs to be minimized to obtain the estimated value $\hat{\beta}_{j}$ is:

$$
J\left(\beta_{0}, \beta_{1}, \cdots, \beta_{m}\right)=\sum_{i=1}^{n}\left(y_{i}-\sum_{j=1}^{m} \beta_{j} x_{i j}\right)^{2}
$$

At present, the commonly used regression methods in trajectory prediction include locally weighted linear regression, locally weighted polynomial regression, and so on. For example, Lee et al. [57] took the aircraft type, aircraft ground speed, altitude, and meteorological data as the input of the model and used the stepwise regression method to systematically determine the inputs and functions of the inputs that are included in the prediction model based on the explanatory power when predicting the arrival time of the aircraft. Hamed et al. [41] used a standard point-mass model and statistical regression method to predict the altitude of climbing aircraft. In addition to the standard linear regression model, two common non-linear regression methods, neural networks, and Loess were used, with principal component analysis to reduce the dimensionality as the input data of the regression methods. Tastambekov et al. [58] considered data preprocessing, localizing, and solving linear regression using wavelet decomposition and then established a local linear regression model for trajectory prediction based on historical radar trajectory data, without using any physical or aviation parameters. Kanneganti et al. [59] used a relatively simple linear prediction model to predict the future position of the aircraft ten minutes in advance by using the aircraft heading and horizontal speed information. Hong et al. [60] combined two machine learning algorithms, a clustering algorithm and regression model. First, the historical trajectory data were clustered to find the main pattern of the trajectory, and then a multi-linear regression model of flight time was constructed for each pattern. A new method was based on the mode information of the previous aircraft to determine the trajectory mode used by the target aircraft in the future, and then the regression prediction model was used under this mode to make predictions.

\subsubsection{Neural Network}

Since a neural network can approximate arbitrary continuous mapping very well, it is a good improvement method compared with general linear regression. Only the standard network structure is introduced here. The standard structure consists of an input layer, a hidden layer with an activation function, and an output layer. When the input is a sample 
$\left\{x_{i}\right\}_{i=1}^{N}$ containing $N$ features (time series containing information such as position and heading), the network output is:

$$
y=\sum_{j=1}^{M} a_{j} f_{j}\left(\sum_{i=1}^{N} w_{j i} x_{i}+b_{i}\right)+c_{j}
$$

$w_{j i}$ and $b_{i}$ are the bias and weight of the hidden layer, $a_{j}$ and $c_{j}$ are the bias and weight of the output layer. The neuron activation functions of the same layer are usually the same. Common activation functions include sigmoid, tanh, and ReLU functions. The network obtains the parameter set $\theta$ by minimizing the prediction error of the data set. Here, the mean square error is selected, that is, when a sample $\left\{y_{k}, x_{1 k}, \cdots, x_{N k}\right\}$ is given, the loss function to be minimized is shown in Equation (16), which refers to the error between the predicted value and the actual value of the track:

$$
\theta=\underset{w_{j i}, b_{i}, a_{j}, c_{j} k=1}{\operatorname{argmin}} \sum_{j=1}^{K}\left(y_{k}-\sum_{j=1}^{M}\left(a_{j} f_{j}\left(\sum_{i=1}^{N} w_{j i} x_{i}+b_{i}\right)+c_{j}\right)\right)^{2}
$$

The most widely used algorithm for solving the optimal parameters is the gradient descent method, which is effectively calculated mainly through the gradient backpropagation of the loss function. This method usually requires many iterations to reach the optimal value. Its common improved algorithms include the Adam algorithm and RMSprop algorithm.

At present, more and more researchers use neural networks to deal with track prediction problems [8,40,61-67]. Commonly used methods include BP neural networks, long short-term memory neural networks (LSTMs), and depth neural networks (DNNs). Neural networks usually take aircraft position and related information as input features and output the probability distribution of the three-dimensional position, estimated flight time, or trajectory at multiple points in the future. Fablec et al. [61] used artificial neural networks to solve the problem of vertical plane trajectory prediction in the two cases of trajectory prediction considering real points during aircraft flight and trajectory generation before aircraft take-off. Hang et al. [40] proposed to establish a hybrid model of DNN and LSTM and used DNN single-step prediction for the correction of LSTM multi-step prediction. Aiming at the problem of weather-related aircraft trajectory prediction, Pang et al. [68] proposed a new conditional generation confrontation network method and used convolutional layers to extract weather features. Pang et al. [69] used a Bayesian neural network for probabilistic trajectory prediction, implemented by using Dropout as Bayesian approximate variational inference in conventional neural networks, and finally output the predicted trajectory with a confidence interval. Wu et al. [70] first used agglomerative hierarchical clustering and a K-means clustering algorithm to cluster the total flight time of the tracks to obtain a central value of flight time fluctuations and then normalized the total flight time of all tracks. The trajectory was then treated as a multivariate time series, and a BP neural network prediction model was trained.

The long short-term memory (LSTM) network can effectively capture the long-term correlation of the sequence and has been successfully applied to various time series prediction tasks in recent years, so the most commonly used neural network in track prediction is the LSTM network. Shi et al. [62] proposed a trajectory prediction model based on the LSTM network, which takes into account the correlation of the adjacent states of the trajectory sequence, which helps to improve the prediction accuracy. Xu et al. [63] proposed a multi-machine trajectory cooperative prediction model based on social long short-term memory (S-LSTM) network. The model builds an LSTM network and a pooling layer for each plane to integrate the hidden states of related planes to effectively capture the interactions between them. Zeng et al. [64] described the 4D trajectory prediction problem as a sequence-to-sequence learning problem and proposed a sequence-to-sequence deep long short-term memory network (SS-DLSTM) for trajectory prediction, which can capture trajectories efficiently with long- and short-time dependencies and repeatability. Ma et al. [71] 
used the LSTM method to exploit the correlation and dependence between the aircraft gliding motion position sequences. Combined with the change in the ground plane motion state, an attenuation memory window is introduced to improve the hidden layer structure and further improve the prediction accuracy of the LSTM model. Zhao et al. [72] proposed a deep long short-term memory (D-LSTM) neural network for aircraft trajectory prediction, which integrated the multi-dimensional features of aircraft trajectories into the LSTM to improve the prediction accuracy of aircraft in complex flight environments.

\subsubsection{Other Methods}

In addition to the two commonly used methods of regression model and neural network, other machine learning methods have also appeared [73-79], such as genetic algorithm (GA), ant colony algorithm, and support vector machine (SVM), etc. Here, this is regarded as a separate category.

In addition, current trajectory prediction also uses clustering algorithms [80-83], such as K-means, density-based clustering, etc., and usually designs appropriate trajectory similarity metrics to improve the clustering effect. Tang et al. [80] proposed an adaptive clustering method that combines the time deviation edit distance trajectory similarity measurement index with the K-means algorithm to improve the accuracy of the nominal flight profile. To improve the accuracy of prediction tasks, the combination of clustering and machine learning prediction methods can significantly improve the prediction accuracy of large-scale clusterable data sets. Therefore, the application of machine learning and clustering to track prediction is a valuable and meaningful research topic [84,85]. For example Barratt et al. [82] studied a probabilistic trajectory generation model in the terminal airspace, first using K-means to cluster the trajectory, and then constructing a Gaussian mixture model from the clustering to achieve accurate trajectory inference. Gallego et al. [84]. analyzed the influence of various operational factors on the vertical profile of the flight trajectory. Firstly, these factors are identified a priori by using a multi-level linear model, and then the influence of these factors on the vertical profile of the flight trajectory is analyzed using two trajectory predictors, the point mass model and the artificial neural network. Le et al. [83] proposed a sector-based short-term trajectory prediction method, which divided multiple trajectory clusters according to the spatial behavior of the historical trajectory in the sector, and used the random forest algorithm to train the corresponding prediction model. Wang et al. [8] used density-based spatial clustering of applications with noise (DBSCAN) method to cluster trajectories into partitions and noises and then used a three-layer neural network to train a prediction model for each type of trajectory.

Table 2. Overview of machine learning model methods.

\begin{tabular}{cc}
\hline & Linear regression: [41,59,60] \\
Segression model & Nopwise regression: [57] \\
& Feedforward neural networks: [61,70,75,84] \\
Elman neural network: [78] \\
LSTM: [62-65,67,71,72] \\
DNN + LSTM: [40] \\
CNN + LSTM: [66] \\
GRU: [79] \\
Neural network model \\
Bayesian neural network: [40,69] \\
Generative adversarial network: [68] \\
\hline Other methods & A gaussian mixture model with clustering: [82] \\
Random forest with clustering: [83] \\
Neural Networks with clustering: [8] \\
Nonparametric interval prediction: [73] \\
Genetic programming: [76]
\end{tabular}




\section{Evaluation Index}

The Euclidean error $(E E)$, the along-track error $(A T E)$, the cross-track error $(C T E)$, and the altitude error $(A E)$ are four widely used metrics to evaluate the performance of prediction methods $[18,64]$. Let $\left(y_{1}^{t}, y_{2}^{t}, y_{3}^{t}\right)$ and $\left(\bar{y}_{1}^{t}, \bar{y}_{2}^{t}, \bar{y}_{3}^{t}\right)$ be the actual 3D position and the estimated 3D position at the timestamp $t$.

The $E E$ measures the difference between the actual and predicted aircraft positions in 3D space:

$$
E E=\frac{1}{T} \sum_{t=1}^{T} \sqrt{\left(\bar{y}_{1}^{t}-y_{1}^{t}\right)^{2}+\left(\bar{y}_{2}^{t}-y_{2}^{t}\right)^{2}+\left(\bar{y}_{3}^{t}-y_{3}^{t}\right)^{2}}
$$

where $T$ is the length of look-ahead time;

The ATE measures the horizontal error along-track:

$$
A T E=\frac{1}{T} \sum_{t=1}^{T}\left[\left(\bar{y}_{1}^{t}-y_{1}^{t}\right) \sin \left(\theta_{t}^{i}\right)+\left(\bar{y}_{2}^{t}-y_{2}^{t}\right) \cos \left(\theta_{t}^{i}\right)\right]
$$

where $\theta_{t}$ denotes the course from north at timestamp $t$;

The CTE measures the horizontal error perpendicular to the nominal track:

$$
C T E=\frac{1}{T} \sum_{t=1}^{T}\left[\left(\bar{y}_{1}^{t}-y_{1}^{t}\right) \cos \left(\theta_{t}\right)-\left(\bar{y}_{2}^{t}-y_{2}^{t}\right) \sin \left(\theta_{t}\right)\right]
$$

The $A E$ is the difference in the vertical positions between the actual and predicted trajectories.

$$
A E=\frac{1}{T} \sqrt{\sum_{t=1}^{T}\left(\bar{y}_{3}^{t}-y_{3}^{t}\right)^{2}}
$$

\section{Open Database}

To provide a comprehensive reference for researchers in related fields, this article introduces the optional databases in trajectory prediction research, which mainly include aircraft performance data, aircraft monitoring data, and meteorological data.

\subsection{Aircraft Performance Data}

Aircraft performance data include the flight envelope (maximum speed, minimum speed, etc.) of each aircraft model, aerodynamics (wing area and drag coefficient), engine thrust and fuel consumption, and other parameters [44]. Currently, available performance databases mainly include the Base of Aircraft Data (BADA), Aircraft Noise and Performance (ANP), etc., of the European Control Center.

\subsubsection{BADA}

BADA is an aircraft performance model developed and maintained by Eurocontrol in cooperation with aircraft manufacturers and operating airlines [52,86]. It is based on the kinetic method for aircraft performance modeling, which can accurately predict the trajectory of the aircraft and related fuel consumption. BADA not only provides a model description of the theoretical basis for calculating aircraft performance parameters but also includes a data set of aircraft-specific coefficients required to calculate aircraft trajectories. The BADA 3 series is today's industry standard for aircraft performance modeling in the nominal part of the flight envelope and provides nearly $100 \%$ coverage of aircraft types operating in Europe. The latest BADA 4 series has improved the accuracy of aircraft performance parameters throughout the flight range, covering $70 \%$ of the aircraft types in Europe. According to the best aircraft performance reference data available, BADA can truly reproduce the geometry, kinematics, and dynamics aspects of aircraft behavior throughout the entire operational flight envelope and all flight phases. Aircraft performance 
models are designed to simulate and predict aircraft trajectories, helping ATM research and operations.

\subsubsection{ANP}

The ANP database is jointly maintained by the US Department of Transportation, the European Control Center, and the European Aviation Safety Agency. It provides the noise and performance characteristics of more than 150 civil aircraft types and is used to calculate noise contours around civil airports. Aircraft manufacturers provide ANP data sets for specific airframe engine types in accordance with the specifications established by the International Civil Aviation Organization and European institutions. The European Aviation Safety Agency is responsible for collecting, verifying, and publishing aircraft ANP data within the scope of Regulation (EU) 598/2014.

\subsection{Aircraft Surveillance Data}

The monitoring data include the current position and speed of the aircraft and provide the real-time status of the aircraft. These data are mainly used to monitor and update the trajectory, such as automatic dependent surveillance broad (ADS-B) data and secondary radar surveillance data.

\subsubsection{Flightradar24}

Flightradar24 is a global flight tracking service that can display real-time air traffic flow from all over the world. It combines data from multiple data sources, including ADS-B, multi-point positioning, and radar data, where ADS-B is the main technology used to receive flight information. Flightradar24 has more than 20,000 ADS-B receiver networks worldwide, which receive flight information from aircraft with ADS-B transponders and send it to the server. Flightradar24 tracks more than 180,000 flights from more than 1200 airlines, traveling to and from more than 4000 airports worldwide in real-time.

\subsubsection{FlightAware}

FlightAware is a digital aviation technology company that operates the world's largest flight tracking and data platform. Relying on global connectivity with various aviation sectors, FlightAware provides global flight tracking solutions, forecasting technology, analysis, and decision-making tools for more than 10,000 aircraft operators and service providers and more than 13,000,000 passengers. FlightAware integrates data from thousands of sources around the world to provide the most accurate and comprehensive flight tracking, including the air traffic control system, ADS-B ground station network, data link reception data of major providers, airline flight information, etc.

\subsubsection{VariFlight Global Flight Tracking Radar}

VariFlight Global Flight Real-time Tracking Radar provides users with real-time tracking of aircraft, aircraft flight trajectory playback, flight trajectory data download, flight status, application for ADS-B equipment, and aircraft picture display information services.

\subsubsection{The OpenSky Network}

The main purpose of the OpenSky network is to improve the safety, reliability, and efficiency of air space use by opening access to real-world air traffic control data to the public. The OpenSky network consists of multiple sensors connected to the Internet by volunteers, industry supporters, and academic/government organizations. It archives all the collected raw data in a large historical database. The database mainly provides data support for researchers from different fields to analyze and improve air traffic control technologies and processes. The OpenSky Network collects raw air traffic control communication, can monitor ADS-B data and S-mode radar data, and recently began to collect flare data. The data attributes it collects include the date, time, horizontal position, altitude, speed, and heading of the aircraft. The takeoff and arrival of the aircraft can be inferred from the 
existing information. The database has the most comprehensive data in the United States and Europe, but unfortunately, it does not have any data before 2013.

\subsection{Meteorological Data}

Meteorological data provide information related to environmental conditions, such as temperature, wind direction and speed, air pressure, and changes in gravity and magnetic force. Currently, commonly used weather databases include EUROCONTROL for Medium-Range Weather Forecasts (ECMWF), North American Mesoscale Forecast System (NAM), and so on. When environmental information is not available, estimated values are sometimes used instead.

\subsubsection{The China Meteorological Data Network}

The China Meteorological Data Network is an upgraded system of the meteorological science data-sharing network, an important part of the national science and technology basic condition platform, the main portal application system of the meteorological cloud, and the unified shared service platform of the China Meteorological Administration that opens authoritative meteorological data resources to domestic and global users and is the data support platform for opening up our country's meteorological service market, promoting the sharing and efficient application of meteorological information resources, and building a new type of meteorological service system. This networks relies on the Comprehensive Meteorological Information Sharing Platform of the China Meteorological Administration to unify the data environment and provide public welfare, equality, and inclusive meteorological big data services to the society and the public. The service methods include Web online services, meteorological data API interface services, and mobile APPs.

\subsubsection{ECMWF}

ECMWF is an independent intergovernmental organization that re-analyzes meteorological data, providing medium-range, monthly, and seasonal weather forecasts, and it is committed to scientific and technological research on the development of numerical models and data assimilation systems, providing Copernicus atmospheric monitoring and climate change services on behalf of the European Community. The data provided are mainly in GRIB and NC formats.

\subsubsection{National Environmental Information Center}

The National Center for Environmental Information (NCEI) provides national and global weather, water, climate, and space weather guidance, forecasts, warnings, and analysis to partners and external user communities. NCEI is one of the most important environmental data archives in the world, having more than 37 petabytes of environmental data. It also includes weather forecast models such as NAM and Global Forecast System for generating weather forecasts.

\subsubsection{Relay of Aircraft Meteorological Data}

Aircraft Meteorological Data Relay (AMDAR) is a component system of the World Meteorological Organization's integrated global observation system, which provides aircraftbased observations for the World Meteorological Observation Program. The AMDAR system mainly uses existing aircraft onboard sensors, computers, and communication systems to collect, process, and format meteorological data and transmit them to the ground station via satellite or radio links. Once the data reach the ground, they are relayed to the National Meteorological and Hydrological Department, processed, quality controlled, and transmitted on the World Meteorological Organization information system. The AMDAR observing system takes more than 700,000 high-quality observations of air temperature, wind speed, and wind direction every day while providing the required position and time information, and more and more humidity and turbulence measurements. The collected data can be used in a variety of meteorological applications, including public weather 
forecasts, climate monitoring, and forecasting, weather disaster warning systems, and, importantly, weather monitoring and forecasting to support the aviation industry.

\subsubsection{WorldClim}

WorldClim is a database of global weather and climate data with high spatial resolution, which has 19 types of global bioclimatic data sets and monthly basic climate data sets.

\section{Challenges and Future Research}

This article introduces the problem of trajectory prediction from the perspective of trajectory prediction process, prediction model, prediction application, and open databases and summarizes the challenges and directions for further research:

(1) The performance of the trajectory prediction model is closely related to the accuracy of information such as aircraft performance parameters, aircraft intent, and meteorological conditions. These input parameters are more or less in error, and small errors in some parameters can lead to catastrophic prediction results. In order to make more accurate predictions, it is possible to strengthen the real-time sharing and transmission of data such as uncertainty, which is a hotspot of current research; in addition, a more robust prediction model can be established through a method research, which is the focus of future research.

(2) In recent years, ensemble learning is a type of machine learning method that uses multiple models or learners for modeling and uses certain rules to integrate the learning results, so as to obtain a machine learning method that is better than a single model or learner. The existing prediction models have their own advantages and disadvantages, and the application scenarios are different. Therefore, integrating different models to build a track prediction fusion model will improve the accuracy and stability of the model.

(3) In general, air traffic congestion on an aircraft's planned route affects the flight path. At the same time, aircraft passing through the same route or waypoint will also affect each other. How to fully consider the overall traffic congestion and the interaction between aircraft when building a prediction model will help improve the accuracy of track prediction.

(4) Probabilistic trajectory prediction is often more practical than deterministic trajectory prediction. The performance of many air traffic intelligent decision-making systems depends on the accuracy of trajectory prediction. However, trajectory prediction is often affected by a variety of factors, resulting in errors in the prediction results of deterministic models. Therefore, in some application scenarios, it is often more reasonable to predict the spatiotemporal distribution of the track.

(5) Most of the research and development of decision support tools are mainly focused on the terminal airspace. The effective operation of these automated decision support systems depends on the results of aircraft trajectory prediction with high reliability and accuracy. However, the complex structure of the airport terminal airspace, the high density of flight flow, and the frequent changes of aircraft flight attitudes bring challenges to the high-precision and reliable prediction of flight paths.

Author Contributions: The authors contributed to the manuscript equally. All authors have read and agreed to the published version of the manuscript.

Funding: This paper is supported by State Key Laboratory of Air Traffic Management System and Technology, Nanjing 210007, China; (NO: SKLATM202007).

Institutional Review Board Statement: Not applicable.

Informed Consent Statement: Not applicable.

Data Availability Statement: This study did not report any data.

Conflicts of Interest: The authors declare no conflict of interest. 


\section{References}

1. Schulz, E.; Airbus. Global Networks, Global Citizens. Global Market Forecast 2018-2037; Airbus, Ed.; Airbus: Blagnac Cedex, France, 2018.

2. Ruiz, S.; Leones, J.L.; Ranieri, A. A novel performance framework and methodology to analyze the impact of 4D trajectory based operations in the future air traffic management system. J. Adv. Transport. 2018, 2018, 1601295. [CrossRef]

3. Song, Y.; Cheng, P.; Mu, C. An Improved Trajectory Prediction Algorithm Based on Trajectory Data Mining for Air Traffic Management. In Proceedings of the 2012 IEEE International Conference on Information and Automation, Shenyang, China, 6-8 June 2012.

4. Brudnicki, D.J.; Kirk, D.B. Trajectory modeling for automated en route air traffic control (AERA). In Proceedings of the 1995 American Control Conference-ACC'95, Seattle, WA, USA, 21-23 June 1995.

5. FAA/EUROCONTROL. Common TP Structure and Terminology in Support of SESAR \& NextGen; Techincal Report FAA/EUROCONTROL: Brussels, Belgium, 2010.

6. Garcia-Chico, J.; Vivona, R.; Cate, K. Characterizing intent maneuvers from operational data: Step towards trajectory prediction uncertainty estimation. In Proceedings of the AIAA Guidance, Navigation and Control Conference and Exhibit, Honolulu, HI, USA, 18-21 August 2008.

7. ICAO. Global Air Traffic Management Operational Concept; ICAO: Montreal, QC, Canada, 2005.

8. Wang, Z.; Liang, M.; Delahaye, D. Short-Term 4d Trajectory Prediction Using Machine Learning Methods. In Proceedings of the SESAR Innovation Day SID, Belgrade, Serbia, 28-30 November 2017.

9. Chatterji, G. Short-Term Trajectory Prediction Methods. In Proceedings of the Guidance, Navigation, and Control Conference and Exhibit, Portland, OR, USA, 9-11 August 1999.

10. Kuchar, J.K.; Yang, L.C. A review of conflict detection and resolution modeling methods. IEEE Trans. Intell. Transp. Syst. 2000, 1, 179-189. [CrossRef]

11. Radanovic, M.; Eroles, M.A.P.; Koca, T.; Gonzalez, J.J.R. Surrounding traffic complexity analysis for efficient and stable conflict resolution. Transp. Res. Part C Emerg. Technol. 2018, 95, 105-124. [CrossRef]

12. Zhang, J.; Liu, J.; Hu, R.; Zhu, H. Online four dimensional trajectory prediction method based on aircraft intent updating. Aerosp. Sci. Technol. 2018, 77, 774-787. [CrossRef]

13. Bronsvoort, J. Contributions to Trajectory Prediction Theory and Its Application to Arrival Management for Air Traffic Control. Ph.D. Thesis, Universidad Politécnica de Madrid, Madrid, Spain, 8 October 2014.

14. Lymperopoulos, I.; Lygeros, J.; Lecchini, A. Model Based Aircraft Trajectory Prediction during Takeoff. In Proceedings of the AIAA Guidance, Navigation, and Control Conference and Exhibit, Keystone, CO, USA, 21-24 August 2006.

15. Porretta, M.; Dupuy, M.D.; Schuster, W.; Majumdar, A.; Ochieng, W. Performance evaluation of a novel 4D trajectory prediction model for civil aircraft. J. Navig. 2008, 61, 393-420. [CrossRef]

16. Schuster, W.; Ochieng, W.; Porretta, M. High-Performance Trajectory Prediction for Civil Aircraft. In Proceedings of the 29th Digital Avionics Systems Conference, Salt Lake City, UT, USA, 3-7 October 2010.

17. Schultz, C.; Thipphavong, D.; Erzberger, H. Adaptive Trajectory Prediction Algorithm for Climbing Flights. In Proceedings of the AIAA Guidance, Navigation, and Control Conference, Minneapolis, MN, USA, 13-16 August 2012.

18. Schuster, W.; Porretta, M.; Ochieng, W. High-accuracy four-dimensional trajectory prediction for civil aircraft. Aeronaut. J. 2012, 116, 45-66. [CrossRef]

19. Benavides, J.V.; Kaneshige, J.; Sharma, S.; Panda, R.; Steglinski, M. Implementation of a Trajectory Prediction Function for Trajectory Based Operations. In Proceedings of the AIAA Atmospheric Flight Mechanics Conference, National Harbor, MD, USA, 13-17 January 2014.

20. Dalmau, R.; Pérez-Batlle, M.; Prats, X. Real-Time Identification of Guidance Modes in Aircraft Descents Using Surveillace Data. In Proceedings of the 2018 IEEE/AIAA 37th Digital Avionics Systems Conference (DASC), London, UK, 23-27 September 2018.

21. Dalmau, R.; Prats, X.; Verhoeven, R.; Bussink, F.; Heesbeen, B. Comparison of various guidance strategies to achieve time constraints in optimal descents. J. Guid. Control. Dyn. 2019, 42, 1612-1621. [CrossRef]

22. Avanzini, G. Frenet-based algorithm for trajectory prediction. J. Guid. Control. Dyn. 2004, 27, 127-135. [CrossRef]

23. Lymperopoulos, I.; Lygeros, J. Sequential Monte Carlo methods for multi-aircraft trajectory prediction in air traffic management. Int. J. Adapt. Control Signal Process. 2010, 24, 830-849. [CrossRef]

24. Lin, Y.; Zhang, J.-W.; Liu, H. An algorithm for trajectory prediction of flight plan based on relative motion between positions. Front. Inf. Technol. Electron. Eng. 2018, 19, 905-916. [CrossRef]

25. Choi, P.P.; Hebert, M. Learning and predicting moving object trajectory: A piecewise trajectory segment approach. Robot. Inst. 2006, 337, 1-17.

26. Ayhan, S.; Samet, H. Aircraft Trajectory Prediction Made Easy with Predictive Analytics. In Proceedings of the 22nd ACM SIGKDD International Conference on Knowledge Discovery and Data Mining, San Francisco, CA, USA, 13-17 August 2016.

27. Lin, Y.; Yang, B.; Zhang, J.; Liu, H. Approach for 4-d trajectory management based on HMM and trajectory similarity. J. Mar. Sci. Technol. 2019, 27, 246-256.

28. Rezaie, R.; Li, X.R. Trajectory Modeling and Prediction with Waypoint Information Using a Conditionally Markov Sequence. In Proceedings of the 2018 56th Annual Allerton Conference on Communication, Control, and Computing (Allerton), Monticello, IL, USA, 2-5 October 2018. 
29. Bar-Shalom, Y.; Li, X.R.; Kirubarajan, T. Estimation with Applications to Tracking and Navigation: Theory Algorithms and Software; John Wiley \& Sons: Hoboken, NJ, USA, 2004.

30. Liu, W.; Hwang, I. Probabilistic trajectory prediction and conflict detection for air traffic control. J. Guid. Control. Dyn. 2011, 34, 1779-1789. [CrossRef]

31. Seah, C.E.; Hwang, I. A Hybrid Estimation Algorithm for Terminal-Area Aircraft Tracking. In Proceedings of the AIAA Guidance, Navigation and Control Conference and Exhibit, Hilton Head, SC, USA, 20-23 August 2007.

32. Seah, C.E.; Hwang, I. Terminal-area aircraft tracking using hybrid estimation. J. Guid. Control. Dyn. 2009, 32, 836-849. [CrossRef]

33. Hwang, I.; Seah, C.E. Intent-based probabilistic conflict detection for the next generation air transportation system. Proc. IEEE 2008, 96, 2040-2059. [CrossRef]

34. Chen, Y.; Cheng, Z.; Wen, S.-L. Modified IMM algorithm for unmatched dynamic models. Syst. Eng. Electron. 2011, 33, $2593-2597$.

35. Jun-Feng, Z.; Xiao-Guang, W.; Fei, W. Aircraft trajectory prediction based on modified interacting multiple model algorithm. J. Donghua Univ. 2015, 32, 180-184.

36. Yepes, J.L.; Hwang, I.; Rotea, M. New algorithms for aircraft intent inference and trajectory prediction. J. Guid. Control. Dyn. 2007, 30, 370-382. [CrossRef]

37. Xi, L.; Jun, Z.; Yanbo, Z.; Wei, L. Simulation Study of Algorithms for Aircraft Trajectory Prediction Based on ADS-B Technology. In Proceedings of the 2008 Asia Simulation Conference-7th International Conference on System Simulation and Scientific Computing, Beijing, China, 10-12 October 2008.

38. Liu, Y.; Li, X.R. Intent based trajectory prediction by multiple model prediction and smoothing. In Proceedings of the AIAA Guidance, Navigation, and Control Conference, Kissimmee, FL, USA, 5-9 January 2015.

39. Choi, H.-C.; Deng, C.; Hwang, I. Hybrid Machine learning and estimation-based flight trajectory prediction in terminal airspace. IEEE Access 2021, 9, 151186-151197. [CrossRef]

40. Zhang, X.; Mahadevan, S. Bayesian neural networks for flight trajectory prediction and safety assessment. Decis. Support Syst. 2020, 131, 113246. [CrossRef]

41. Ghasemi Hamed, M.; Gianazza, D.; Serrurier, M.; Durand, N. Statistical prediction of aircraft trajectory: Regression methods vs point-mass model. In Proceedings of the ATM Seminar, Chicago, IL, USA, 10-13 June 2013.

42. Weitz, L.A. Derivation of a Point-Mass Aircraft Model Used for Fast-Time Simulation; MITRE Corporation: Bedford, MA, USA, 2015.

43. Schuster, W. Trajectory prediction for future air traffic management-Complex manoeuvres and taxiing. Aeronaut. J. 2015, 119, 121-143. [CrossRef]

44. Fukuda, Y.; Shirakawa, M.; Senoguchi, A. Development and evaluation of trajectory prediction model. In Proceedings of the 27th International Congress of the Aeronautical Sciences, Nice, France, 19-24 September 2010.

45. Tang, X.-M.; Han, Y.-X. 4D trajectory estimation for air traffic control automation system based on hybrid system theory. Promet Traffic Transp. 2012, 24, 91-98. [CrossRef]

46. Lee, J.; Lee, S.; Hwang, I. Hybrid system modeling and estimation for arrival time prediction in terminal airspace. J. Guid. Control. Dyn. 2016, 39, 903-910. [CrossRef]

47. Felix, F.N.; Ruiz, M.V.; Querejeta, C.; Gallo, E.; Leones, J.L. Predicting Aircraft Trajectory. US9250099B2 Patents, 2 February 2016.

48. Lopez Leones, J. Definition of an aircraft intent description language for air traffic management applications. Ph.D. Thesis, University of Glasgow, Glasgow, UK, 2008.

49. López-Leonés, J.; Vilaplana, M.A.; Gallo, E.; Navarro, F.A.; Querejeta, C. The aircraft intent description language: A key enabler for air-ground synchronization in trajectory-based operations. In Proceedings of the 2007 IEEE/AIAA 26th Digital Avionics Systems Conference, Dallas, TX, USA, 21-25 October 2007.

50. Thipphavong, D.P.; Schultz, C.A.; Lee, A.G.; Chan, S.H. Adaptive algorithm to improve trajectory prediction accuracy of climbing aircraft. J. Guid. Control. Dyn. 2013, 36, 15-24. [CrossRef]

51. Baklacioglu, T.; Cavcar, M. Aero-propulsive modelling for climb and descent trajectory prediction of transport aircraft using genetic algorithms. Aeronaut. J. 2014, 118, 65-79. [CrossRef]

52. Nuic, A.; Poinsot, C.; Iagaru, M.G.; Gallo, E.; Navarro, F.A.; Querejeta, C. Advanced aircraft performance modeling for ATM: Enhancements to the BADA model. In Proceedings of the 24th Digital Avionics System Conference, Washington, DC, USA, 30 September-3 October 2005.

53. Alligier, R.; Gianazza, D.; Durand, N. Learning the aircraft mass and thrust to improve the ground-based trajectory prediction of climbing flights. Transp. Res. Part C Emerg. Technol. 2013, 36, 45-60. [CrossRef]

54. Sun, J.; Ellerbroek, J.; Hoekstra, J.M. WRAP: An open-source kinematic aircraft performance model. Transp. Res. Part C Emerg. Technol. 2019, 98, 118-138. [CrossRef]

55. Sun, J.; Ellerbroek, J.; Hoekstra, J.M. Aircraft initial mass estimation using Bayesian inference method. Transp. Res. Part C Emerg. Technol. 2018, 90, 59-73. [CrossRef]

56. Alligier, R.; Gianazza, D. Learning aircraft operational factors to improve aircraft climb prediction: A large scale multi-airport study. Transp. Res. Part C Emerg. Technol. 2018, 96, 72-95. [CrossRef]

57. De Leege, A.; van Paassen, M.; Mulder, M. A machine learning approach to trajectory prediction. In Proceedings of the AIAA Guidance, Navigation, and Control (GNC) Conference, Boston, MA, USA, 19-22 August 2013.

58. Tastambekov, K.; Puechmorel, S.; Delahaye, D.; Rabut, C. Aircraft trajectory forecasting using local functional regression in Sobolev space. Transp. Res. Part C Emerg. Technol. 2014, 39, 1-22. [CrossRef] 
59. Kanneganti, S.T.; Chilson, P.B.; Huck, R. Visualization and prediction of aircraft trajectory using ADS-B. In Proceedings of the NAECON 2018-IEEE National Aerospace and Electronics Conference, Dayton, OH, USA, 23-26 July 2018.

60. Hong, S.; Lee, K. Trajectory prediction for vectored area navigation arrivals. J. Aerosp. Inf. Syst. 2015, 12, 490-502. [CrossRef]

61. Le Fablec, Y.; Alliot, J.-M. Using neural networks to predict aircraft trajectories. In Proceedings of the IC-AI, Las Vegas, NV, USA, 28 June-1 July 1999.

62. Shi, Z.; Xu, M.; Pan, Q.; Yan, B.; Zhang, H. LSTM-based flight trajectory prediction. In Proceedings of the 2018 International Joint Conference on Neural Networks (IJCNN), Rio de Janeiro, Brazil, 8-13 July 2018.

63. Xu, Z.; Zeng, W.; Chu, X.; Cao, P. Multi-aircraft trajectory collaborative prediction based on social long short-term memory network. Aerospace 2021, 8, 115. [CrossRef]

64. Zeng, W.; Quan, Z.; Zhao, Z.; Xie, C.; Lu, X. A deep learning approach for aircraft trajectory prediction in terminal airspace. IEEE Access 2020, 8, 151250-151266. [CrossRef]

65. Yang, K.; Bi, M.; Liu, Y.; Zhang, Y. LSTM-based deep learning model for civil aircraft position and attitude prediction approach. In Proceedings of the 2019 Chinese Control Conference (CCC), Guangzhou, China, 27-30 July 2019.

66. Ma, L.; Tian, S. A hybrid CNN-LSTM model for aircraft $4 \mathrm{D}$ trajectory prediction. IEEE Access 2020, 8, 134668-134680. [CrossRef]

67. Shi, Z.; Xu, M.; Pan, Q. 4-D flight trajectory prediction with constrained LSTM network. IEEE Trans. Intell. Transp. Syst. 2020, 22, 7242-7255. [CrossRef]

68. Pang, Y.; Liu, Y. Conditional generative adversarial networks (CGAN) for aircraft trajectory prediction considering weather effects. In Proceedings of the AIAA Scitech 2020 Forum, Orlando, FL, USA, 6-10 January 2020.

69. Pang, Y.; Liu, Y. Probabilistic aircraft trajectory prediction considering weather uncertainties using dropout as bayesian approximate variational inference. In Proceedings of the AIAA Scitech 2020 Forum, Orlando, FL, USA, 6-10 January 2020.

70. Wu, Z.-J.; Tian, S.; Ma, L. A 4D trajectory prediction model based on the BP neural network. J. Intell. Syst. 2019, $29,1545-1557$. [CrossRef]

71. Ma, Z.; Yao, M.; Hong, T.; Li, B. Aircraft surface trajectory prediction method based on LSTM with attenuated memory window. J. Phys. Conf. Ser. 2019, 1215, 012003. [CrossRef]

72. Zhao, Z.; Zeng, W.; Quan, Z.; Chen, M.; Yang, Z. Aircraft trajectory prediction using deep long short-term memory networks. In Proceedings of the 19th COTA International Conference of Transportation Professionals CICTP 2019, Nanjing, China, 6-8 July 2019; pp. 124-135.

73. Hamed, M.G. Méthodes Non-Paramétriques pour la Prévision D intervalles avec HAUT niveau de Confiance: Application à la Prévision de Trajectoires D avions. Ph.D. Thesis, Institut National Polytechnique de Toulouse-INPT, Toulouse, France, 2014.

74. Shen, Z.; Tang, X. A novel 4D track prediction approach combining empirical mode decomposition with nonlinear correlation coefficient. In Proceedings of the COTA International Conference of Transportation Professionals CICTP 2015, Beijing, China, 24-27 July 2015; pp. 25-34.

75. Gallego, C.E.V.; Comendador, V.F.G.; Carmona, M.A.A.; Valdés, R.M.A.; Nieto, F.J.S.; Martínez, M.G. A machine learning approach to air traffic interdependency modelling and its application to trajectory prediction. Transp. Res. Part C Emerg. Technol. 2019, 107, 356-386. [CrossRef]

76. Zhang, C.; Zhang, X.; Shi, C.; Liu, W. Aircraft trajectory prediction based on genetic programming. In Proceedings of the 2016 3rd International Conference on Information Science and Control Engineering (ICISCE), Beijing, China, 8-10 July 2016.

77. Chen, Z.; Guo, D.; Lin, Y. A deep gaussian process-based flight trajectory prediction approach and its application on conflict detection. Algorithms 2020, 13, 293. [CrossRef]

78. Min, W.; Jiawei, W.; Jinhui, G.; Lihua, S.; Bogong, A. Multi-point prediction of aircraft motion trajectory based on GA-ElmanRegularization neural network. Integr. Ferroelectr. 2020, 210, 116-127. [CrossRef]

79. Zhang, H.; Huang, C.; Xuan, Y.; Tang, S. Real-time prediction of air combat flight trajectory using GRU. Syst. Eng. Electron. Technol. 2020, 42, 2546-2552.

80. Tang, X.; Chen, P.; Zhang, Y. 4D trajectory estimation based on nominal flight profile extraction and airway meteorological forecast revision. Aerosp. Sci. Technol. 2015, 45, 387-397. [CrossRef]

81. Fernández, E.C.; Cordero, J.M.; Vouros, G.; Pelekis, N.; Kravaris, T.; Georgiou, H.; Ayhan, S. DART: A machine-learning approach to trajectory prediction and demand-capacity balancing. In Proceedings of the SESAR Innovation Days, Belgrade, Serbia, 28-30 November 2017; pp. 28-30.

82. Barratt, S.T.; Kochenderfer, M.J.; Boyd, S.P. Learning probabilistic trajectory models of aircraft in terminal airspace from position data. IEEE Trans. Intell. Transp. Syst. 2018, 20, 3536-3545. [CrossRef]

83. Le, T.H.; Tran, P.N.; Pham, D.T.; Schultz, M.; Alam, S. Short-term trajectory prediction using generative machine learning methods. In Proceedings of the ICRAT 2020 Conference, Tampa, FL, USA, 23-26 June 2020.

84. Gallego, C.E.V.; Comendador, V.F.G.; Nieto, F.J.S.; Imaz, G.O.; Valdés, R.M.A. Analysis of air traffic control operational impact on aircraft vertical profiles supported by machine learning. Transp. Res. Part C Emerg. Technol. 2018, 95, 883-903. [CrossRef]

85. Zeng, W.; Xu, Z.; Cai, Z.; Chu, X.; Lu, X. Aircraft trajectory clustering in terminal airspace based on deep autoencoder and gaussian mixture model. Aerospace 2021, 8, 266. [CrossRef]

86. Nuic, A. User manual for the Base of Aircraft Data (BADA) revision 3.10. Atmosphere 2010, 2010, 001. 